\title{
THE SUPPRESSION OF THE FALSE CODLING MOTH IN SOUTH AFRICA USING AN AW-IPM APPROACH WITH A SIT COMPONENT
}

\author{
N. BOERSMA \\ Xsit (Pty) Ltd., 2 Schalk Patience Street, Citrusdal 7340, South Africa; \\ nb@xsit.co.za
}

\begin{abstract}
SUMMARY
The false codling moth, Thaumatotibia leucotreta (Meyrick) (Lepidoptera: Tortricidae), is native to subSaharan Africa, where it infests various commercial, and wild, fruit-bearing plants. This major pest is not present in the Americas, Europe, and Asia, and therefore has phytosanitary implications, which impose severe limitations on potential South African exports. Consequently, this pest represents a severe threat to the fruit industry of South Africa, in terms of socio-economic impacts on both fruit production and job security. Although the pest can be managed to some extent with insecticides, mating disruption, and orchard sanitation, a long-term environment-friendly solution was needed. This became more evident as $T$. leucotreta developed resistance to available insecticides, while stricter quarantine measures were enforced by importers of African citrus. In 2002, research commenced on an area-wide integrated pest management (AW-IPM) programme in conjunction with the development of the Sterile Insect Technique (SIT) for the false codling moth. Commercial sterile insect releases started in the 2007-2008 season over 1500 ha of citrus orchards in Citrusdal, Western Cape Province, but by 2017-2018 had gradually expanded to almost 19000 ha in three different citrus producing regions of South Africa. The programme is currently owned by the Citrus Growers Association (CGA) that have contributed to the steady growth of the SIT programme in the citrus industry. Over the past ten years the status of $T$. leucotreta as a pest threat was systematically reduced in areas where the SIT was practiced on an area-wide basis, compared to non-release areas.
\end{abstract}

Key Words: Citrus, navel orange, area-wide, Sterile Insect Technique, pest management, Thaumatotibia leucotreta, Tortricidae, resistance, quarantine, South African exports, Western Cape

\section{THE PROBLEM}

The false codling moth Thaumatotibia leucotreta (Meyrick) (Lepidoptera: Tortricidae) is a polyphagous indigenous pest of both cultivated crops and wild plants in sub-Saharan Africa. False codling moth was first noted in the Paarl region of the Development and Field Application, pp. 93-109. CRC Press, Boca Raton, Florida, USA. (C) 2021 IAEA 
Western Cape Province (South Africa) around 1969 (Hofmeyr et al. 2015). Although it attacks many different deciduous, subtropical, and tropical plants, it prefers citrus as one of its primary hosts.

By the mid-1970s T. leucotreta was detected at a holiday resort, $170 \mathrm{~km}$ north of the Paarl region near Citrusdal, an important citrus exporting region in the Western Cape Province (Hofmeyr et al. 2015). By the end of the 1970s it had spread through some parts of the valley, with heavy infestations in navel orange orchards (Hofmeyr et al. 2015).

The presence of this insect represents a high phytosanitary risk for South African fruit exports to the USA, Asia, and Europe. The economic threats imposed by the pest to the fruit growers and the industry of South Africa may also have severe socioeconomic consequences for food and job security. The situation was exacerbated after T. leucotreta developed resistance against available registered insecticides and stricter regulations were imposed on exporters (Hofmeyr and Pringle 1998). This included a zero tolerance for T. leucotreta and the requirement of a post-harvest cold treatment (Hofmeyr et al. 2016a, 2016b).

\section{PRE-OPERATIONAL ACTIVITIES}

Although the pest has been managed to some extent, by integrating control tactics such as insecticides, mating disruption, and orchard sanitation, a longer-term solution was needed. In 2002, research was conducted to develop an area-wide integrated pest management system (AW-IPM programme) with an SIT component (Hendrichs et al. 2007; Klassen and Vreysen 2021). Citrus Research International (CRI) (Pty) Ltd, the Citrus Growers Association of South Africa (CGA), the Joint Food and Agriculture Organization of the United Nations/International Atomic Energy Agency Division (FAO/IAEA), the United States Department of Agriculture (USDA) through its Agricultural Research Service (ARS) and Centre for Plant Health Science and Technology (CPHST), joined resources and efforts to develop and test the efficacy of a SIT programme for T. leucotreta.

During the first phase of research, the radiation biology and inherited sterility of T. leucotreta (Bloem et al. 2003) was investigated, which was followed by field cage trials to evaluate mating compatibility and competitiveness (Hofmeyr et al. 2005). The results of these biological studies on the effect of gender and irradiation dose on T. leucotreta are shown in Fig. 1. As expected, fertility of both male and female moths declined with increasing irradiation dose (Bloem et al. 2003). This dose effect was greater for crosses involving irradiated female moths, which were almost completely sterile when treated with a dose of $200 \mathrm{~Gy}$, while irradiated males still had a residual fertility of 5.2\% when treated with a dose of 350 Gy (Fig. 1) (Bloem et al. 2003). Similar to other Lepidoptera, T. leucotreta exhibited inherited sterility when partially sterile male moths copulated with wild female counterparts (Carpenter et al. 2004). The resulting $F_{1}$ progeny was shown to be fully sterile, mostly male, and took longer to develop (Bloem et al. 2003). Furthermore, the $\mathrm{F}_{1}$ generation would either fail to hatch or would develop into sterile, but fully competitive $F_{1}$ adults, that would provide additional pest population suppression in the subsequent generation. 
The promising results led to the next research phase in the Olifants River Valley, which involved a SIT pilot study in citrus orchards during the 2005-2006 season (Hofmeyr et al. 2015). This involved the release of sterile T. leucotreta adults in a 35 .ha navel orange orchard, surrounded by natural vegetation, while another navel orchard was used as a control.

Releases were performed over a 29-week period, with a total of 2000 sterile moths released per week. Thirteen delta traps evenly spaced over the orchards, equipped with synthetic pheromone (Cardiff Chemicals, Cardiff, UK) in Lorelei dispensers, were used. The goal was to create an overflooding ratio within the orchards and this was maintained at no less than 1 wild: 10 sterile moths per week (Hofmeyr et al. 2005; Hofmeyr et al. 2015). The encouraging results from this pilot study indicated a $77 \%$ decrease of wild $T$. leucotreta trap catches and an approximate $95 \%$ reduction of $T$. leucotreta related fruit infestations, compared to the non-SIT area.

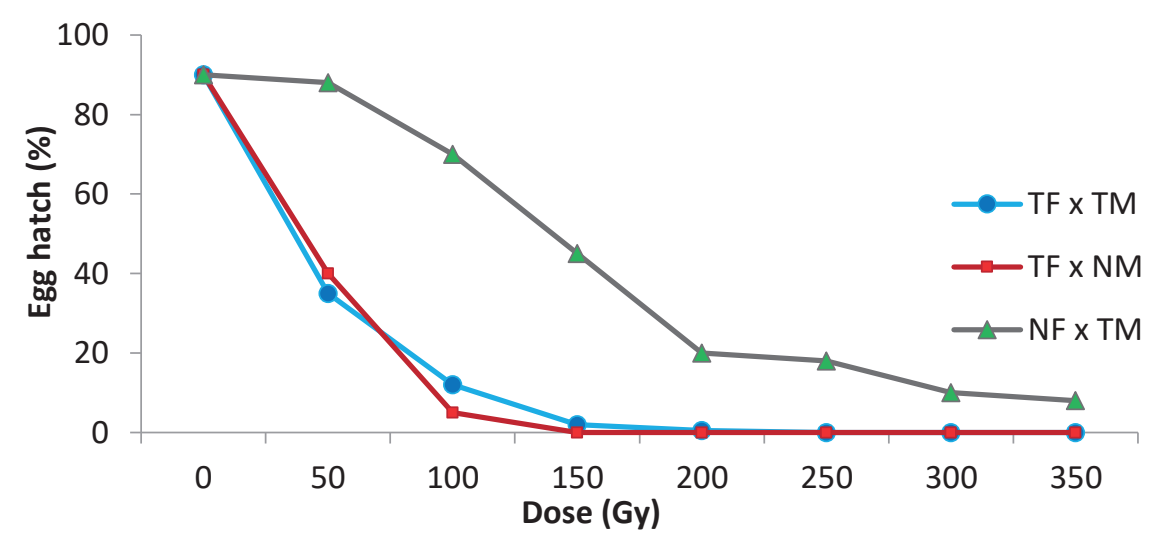

Figure 1. Effect of irradiation dose administered to T. leucotreta adults on the mean egg hatch (\%) per mated female. Males and females were treated (T) with 50, 100, 150, 200, 250, 300 , and 350 Gy and inbred (TF $x$ TM) or out-crossed (TF $x$ NM, NF x TM) to untreated adults (N) (adapted from Bloem et al. 2003).

The results of this 2005-2006 season pilot project in the Citrusdal region are shown in both Figs. 2 and 3. From the results obtained in this initial trial, the South African citrus industry was convinced to fast-track the commercial introduction of the SIT programme for T. leucotreta.

As a result, in 2006, the private company Xsit (Pty) Ltd. was established to manage the production, sterilisation, and the release of sterile T. leucotreta in the Citrusdal region. New equipment was designed to upscale moth production and to replace the insufficient infrastructure being used for the small-scale rearing of $T$. leucotreta (Hofmeyr et al. 2015). The new mass-rearing facility became operational in early 2007, and the release of irradiated moths commenced in November of that year. 


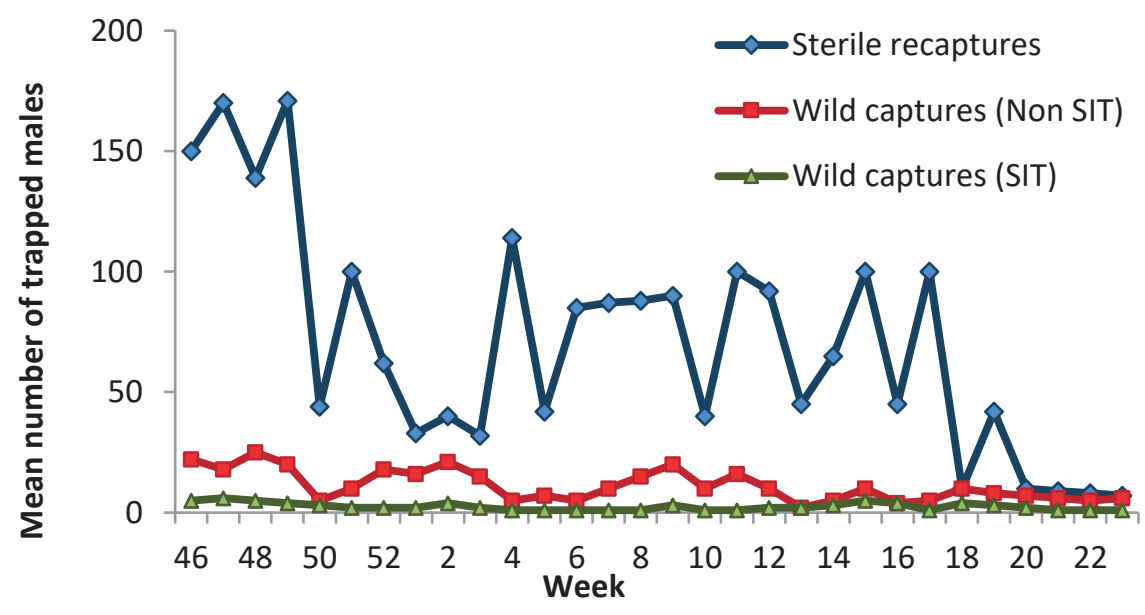

Figure 2. Capture of released (irradiated and topically marked) and wild T. leucotreta males in SIT-treated and non-SIT-treated navel orange orchards as part of a SIT pilot project carried out in the Citrusdal region during the 2005-2006 season. A minimum ratio of 1:10 wild:sterile moths were maintained throughout the pilot trial in the SIT-treated orchard (adapted from Hofmeyr et al. 2015).

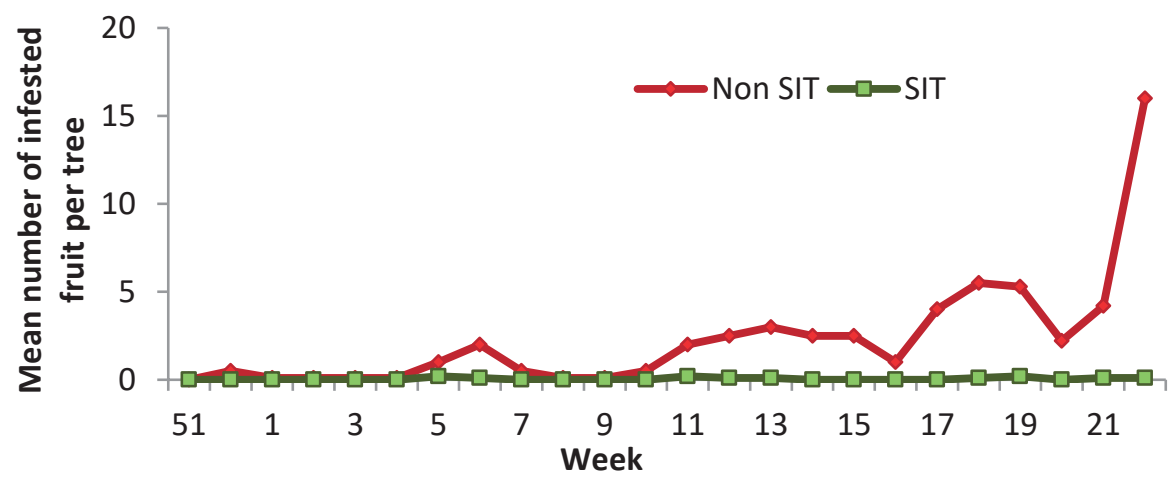

Figure 3. Fruit drop due to T. leucotreta infestation in non-SIT and SIT-treated citrus orchards (35 ha) as part of a SIT pilot project carried out in the Citrusdal region, Western

Cape Province, during the 2005-2006 season.

\section{THE FIRST DAYS OF THE SIT PROGRAMME, EXPANSIONS AND IMPROVEMENTS}

Since the commercialization of the AW-IPM programme with a SIT component in Citrusdal in 2007, many new systems and equipment have been designed, developed, and manufactured in a relatively short time. Production monitoring systems for traceability, cold chain management, and quality management of reared moths were developed and constantly improved. 
From 2007 to 2018 the initial 1500 ha SIT-treated area in the Citrusdal region was gradually increased to 4800 ha by incorporating the rest of the Olifants River Valley. Sterile insect releases are also being carried out over 6500 and 2200 ha of the Sundays River and Gamtoos River valleys in the Eastern Cape Province, respectively. During the 2016-2017 season, the release of sterile T. leucotreta was also expanded to the lower Orange River area in the Northern Cape Province and the Hex River Valley of the Western Cape Province, the latter, an important table grape export region, treating 1500 and 4000 ha respectively. At the time of writing (2018), this privately-owned programme was providing sterile insects on a weekly basis to cover more than 18000 ha.

There was a progressive seasonal improvement in wild T. leucotreta suppression following routine releases of sterile moths in all treated areas. The results showed a reduction in crop losses and fewer rejections of fruit consignments destined for exports due to T. leucotreta presence. While this rapid growth of the programme was very exciting, it was accompanied by many challenges and hardships that sometimes threatened its existence.

\subsection{Rearing Equipment}

During the initial days of programme implementation, some of the old equipment and processes developed for the pilot trial were utilized in the commercial rearing programme. However, with the expansion of the programme, it became apparent that most of these systems were inadequate, and only suitable for small-scale rearing of $T$. leucotreta (Hofmeyr et al. 2015).

\subsubsection{Larval Diet Preparation Equipment}

A new artificial diet (Moore 2002; Moore et al. 2014) was introduced for rearing purposes in 2007. The diet was prepared using large-scale equipment from the baking industry, and then pulsed into $500 \mathrm{ml}$ glass jars fitted with breathable replaceable paper membranes, allowing for gas exchange, in the metallic screw-lids (Hofmeyr et al. 2015). The individual jars were placed by hand into stainless steel baskets, containing 25 rearing jars each. Baskets were then stacked on a steel trolley, holding up to 16 baskets before they were pulled into an oven. Although the oven was an innovative piece of equipment, it was not ideally suited, as evidenced by the uneven cooking of the diet, and lack of sterilisation. After baking two trolleys per oven cycle with 400 glass jars each, they were placed in a room for cooling. Each jar of diet was inoculated with approximately one thousand 24-h old eggs via placement of an egg sheet, sterilised by an $8 \%$ formaldehyde solution, on top of the diet (Hofmeyr et al. 2015).

As the programme expanded and diet preparation increased from 6000 to 20000 bottles a day, the handling and diet preparation processes had to be re-considered. Disadvantages of the jars included relatively high costs, susceptibility to breakage, and requirement for individual handling and cleaning after larvae emerged. In 2013, new technologies to prepare the diet were investigated. These included radiowave, microwave, infrared, steam, and extrusion. After completing the initial trials, only radiowaves, microwaves, and extrusion seemed potentially viable. 
Different cooking times and temperatures were tested to develop a cost-effective method for delivering a diet producing high larval yields without a negative impact on larval development. Although several parameters were tested, the following criteria were critical in validating the optimum cooking/sterilisation process:

1. Proportion of large $5^{\text {th }}$ instar larvae $(0.04 \mathrm{~g} \pm 1$ and $>10 \mathrm{~mm})$ produced.

2. Feed conversion ratio, denoted by the amount of diet required to produce one large $5^{\text {th }}$ instar larva.

3. Absence of viral and bacterial infected larvae - denoted in the amount ( $\mathrm{g}$ ) of diet required to produce one healthy larva.

To validate the correct process and cooking protocol (time vs. temperature), the number of large larvae ( $5^{\text {th }}$ instar) produced was counted. It was evident that both the microwave and the extrusion processes resulted in the highest number of large larvae with the least feed (best feed conversion). The original Xsit diet required $0.35 \mathrm{~g}$ to rear one large larva, while both the microwave and the extrusion required only between $0.21 \mathrm{~g}$ and $0.22 \mathrm{~g}$, respectively to rear one large larva. It was clear that the best results were obtained with the laboratory microwave and the extruder, where approximately $90 \%$ of the reared larvae reached $5^{\text {th }}$ instar on day 12 , while only $40 \%$ of the control diet reached $5^{\text {th }}$ instar at the same time (Fig. 4).

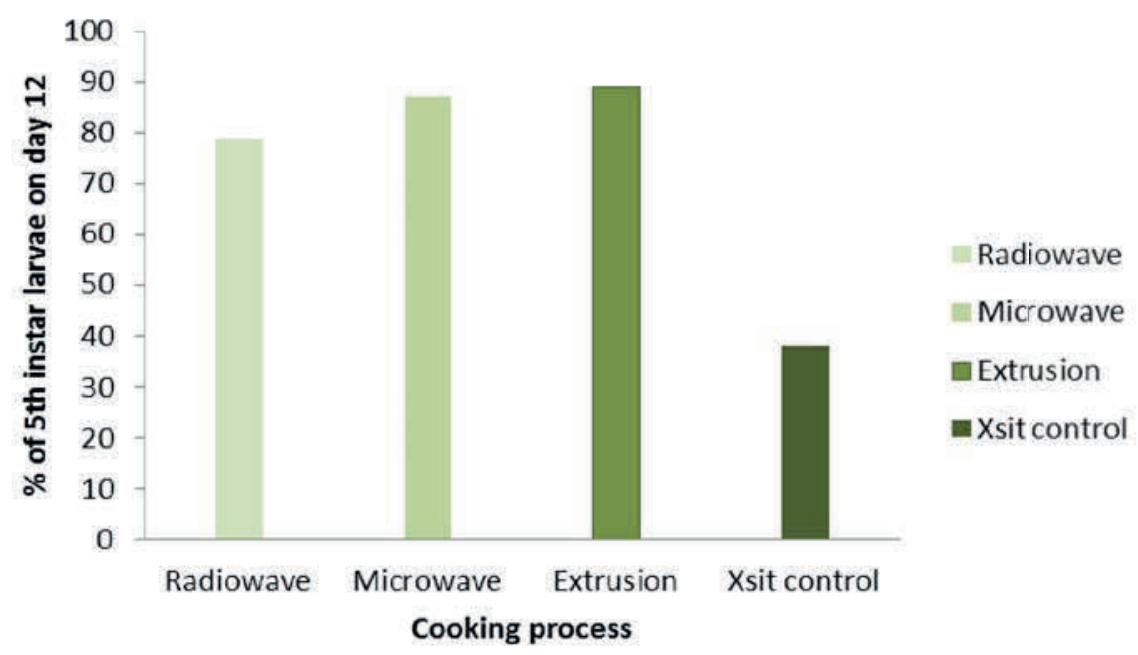

Figure 4. Percentage of large larvae per diet preparation treatment. Results of the preliminary laboratory studies were confirmed in this laboratory trial. Microwave and extrusion samples resulted in the highest amount of $5^{\text {th }}$ instar larvae on day 12.

The next step was to test both processes in a commercial trial. In 2015, several experiments were conducted in a commercial microwave. These trials were repeated three times under different settings, but the positive results obtained with the laboratory microwave $(1000 \mathrm{~W})$ could not be replicated in the commercial microwave. After further investigation in obtaining a commercial microwave, the lack of support of the industry was evident, and it was decided to continue with the 
extrusion process. Commercial trials with different extruder processes and settings were tested until the end of 2016, when the correct settings and consistency of the diet were obtained, with proven results of growth and yield of larvae replicated on a semicommercial scale; nearly $90 \%$ of larvae had grown on the extruded diet, and reached $5^{\text {th }}$ instar on day 12 , compared to only $40 \%$ on the Xsit diet.

Xsit purchased its own extruder plant in July 2017. However, after the commissioning of the extruder, it was clear that the larval diet was not similar or even comparable to the product developed over the past four years. The extruded larval diet was sticky and could hardly be packed or handled, while larval growth was retarded, and yields were low. The larval diet produced was therefore, not fit for use. For six months, extensive trials were carried out, testing all variables, including raw product variability and extruder conditions. It was concluded that the main cause of the problem was (a) the dextrinisation and gelatinisation of the starch in the diet, and (b) the inconsistent granule size of the maize meal, which comprises $80 \%$ of the diet. During the extrusion process the starch granules swell when pressurised under high temperatures during cooking and drying, and then shrink as soon as the product cools down. In the event, when the diet is cooked at a too high temperature, the starch molecules lose the ability to swell, leading them to shear and burst. This will lead to the loss of the semi-crystalline structure of the starch, while the smaller amylose molecules leach from the granule to form new chemical structures which cannot be digested by the larvae. During cooling, the semi-crystalline structure recovers and, provided that the granules did not burst, will re-align to a similar position or structure prior to cooking. This phenomenon is known as retrogradation (Oates 1997; Wang et al. 2015). As the initial research was conducted in different stages, the diet was cooled prior to drying, while at the new commercial plant the extruded diet was immediately transferred to the oven for drying, leading to dextrinisation and gelatinisation of the starch. This assumption was confirmed during trials where the diet was allowed to cool before drying. As a final outcome, excellent results were obtained which were similar to those obtained in the research done over the previous four years. An additional cooling unit was introduced after extrusion, before drying.

\subsubsection{Rearing Containers}

During the original rearing of the larvae in glass jars, the screw lids and membranes were removed when larvae reached $5^{\text {th }}$ instar. The jars were placed on their sides to assist larval exit. An integrated aperture below each basket held the pupation substrate, which is a square of polycarbonate honeycomb material, $570 \mathrm{~mm} \times 530 \mathrm{~mm} \times 11 \mathrm{~mm}$ with $6 \mathrm{~mm}$ diameter aperture, placed on a $570 \mathrm{~mm} \times 530 \mathrm{~mm} \times 3 \mathrm{~mm}$ fibre sheet.

In 2012, the whole false codling moth colony suffered from a severe bacterial infection in the facility. After an intense investigation it appeared that miniscule holes between the honeycomb cells became breeding sites for Bacillus cereus, Gramnegative opportunistic bacteria that undermine the immune system of the larvae, killing them in a matter of days. In response, the polycarbonate sheets were replaced with disposable, pre-manufactured sheets of corrugated, single-face cardboard. 
In parallel with the development of the new diet preparation system described above, a replacement of the glass jars was pursued. Several types of containers were investigated, including paper bags, starch bags, polyethylene cups, and disposable polyethylene bags. The most significant challenge was to find a similar membrane to the one used in the jars. The new extruded diet was more prone to drying out, but one had to keep in mind that the regulation and exchange of gasses were critical. A material with these specific properties, while preserving moisture, had to be found.

In 2016, disposable polyethylene bags $(280 \mathrm{~mm} \times 160 \mathrm{~mm})$, with a breathable polyethylene-based microporous membrane, were introduced. These were automatically filled by a diet dispensing machine. A volume of water equal to $47 \%$ of the total volume of the dry mix was added to form a fluffy diet, while $250 \mathrm{~g}$ of larval diet was required to produce at least 550 larvae per unit. The bags were consequently phased in to replace the glass jars. Egg sheets containing $800 \pm 100$ T. leucotreta eggs, dipped in an $8 \%$ formaldehyde solution to prevent contamination by any bacteria and/or virus, were then placed on the diet and sealed. The bags were then assembled on a rearing cart containing 480 bags. When ready for pupation, usually on day 12 of the rearing cycle, the larvae chew their way out of the bags and descend on silk threads to the cardboard pupation substrate, placed $30 \mathrm{~mm}$ below the bags.

After the implementation of this new system, significant production losses were experienced due to larvae dying in the bags before reaching the $5^{\text {th }}$ instar. Upon investigation it was determined that the HVAC system was not capable of handling the large volume of $\mathrm{CO}_{2}$ generated by the large number of larvae reared per $\mathrm{m}^{2}$. Consequently, a new HVAC system with increased capacity and higher air change rate was designed and installed by the end of the 2016-2017 season to ensure sustainable production of sterile insects on a continuous basis.

\subsubsection{Moth Emergence Cabinets}

The pupation boards were placed into custom designed steel emergence cabinets to permit moth emergence and collection. Each cabinet, $1550 \mathrm{~mm}$ x $630 \mathrm{~mm}$ x $940 \mathrm{~mm}$, was welded on a $900 \mathrm{~mm}$ supporting framework (Fig. 5). The cabinets were divided longitudinally with a perforated stainless-steel sheet separating two compartments: a back compartment, $740 \mathrm{~mm}$ deep with an access door, containing 50 horizontally placed pupation sheets, and a front compartment, $200 \mathrm{~mm}$ deep with a glass door to the outside, allowing moths to move phototactically from the back into the front compartment (Boersma and Carpenter 2016). The front compartment was lightly dusted with talcum powder and was fitted with a collection cone at the bottom, attached to a plenum-based air-braking moth collection system.

Establishing the correct speed of the airstream that transferred the moths from the moth cabinet to the collection room was challenging, as too high airflow resulted in damaged moths, while too low airflow caused clogging of moths. This was resolved by adjusting the airspeed to $12 \mathrm{~m} / \mathrm{s}$ for transferring moths to the collection pans, while reducing the airstream to $3 \mathrm{~m} / \mathrm{s}$ as they enter the collection room, allowing for a soft landing (Hofmeyr and Pretorius 2010). 
Scaling up the release area from 3300 ha to more than 18000 ha induced a lot of pressure on these emergence cabinets, resulting in problems with temperature consistency, clogging of moths due to overcrowding, and production losses due to moths escaping from these old cabinets and equipment. In 2015 cabinets were redesigned, allowing for better airflow by making the following improvements:

1) the steel sheets of the cabinets were folded rather than welded for better durability;

2) extra space was provided at the back to allow increased airflow between pupation boards, resulting in fewer temperature spikes;

3) use of rubber sealed Perspex doors for better sealing.

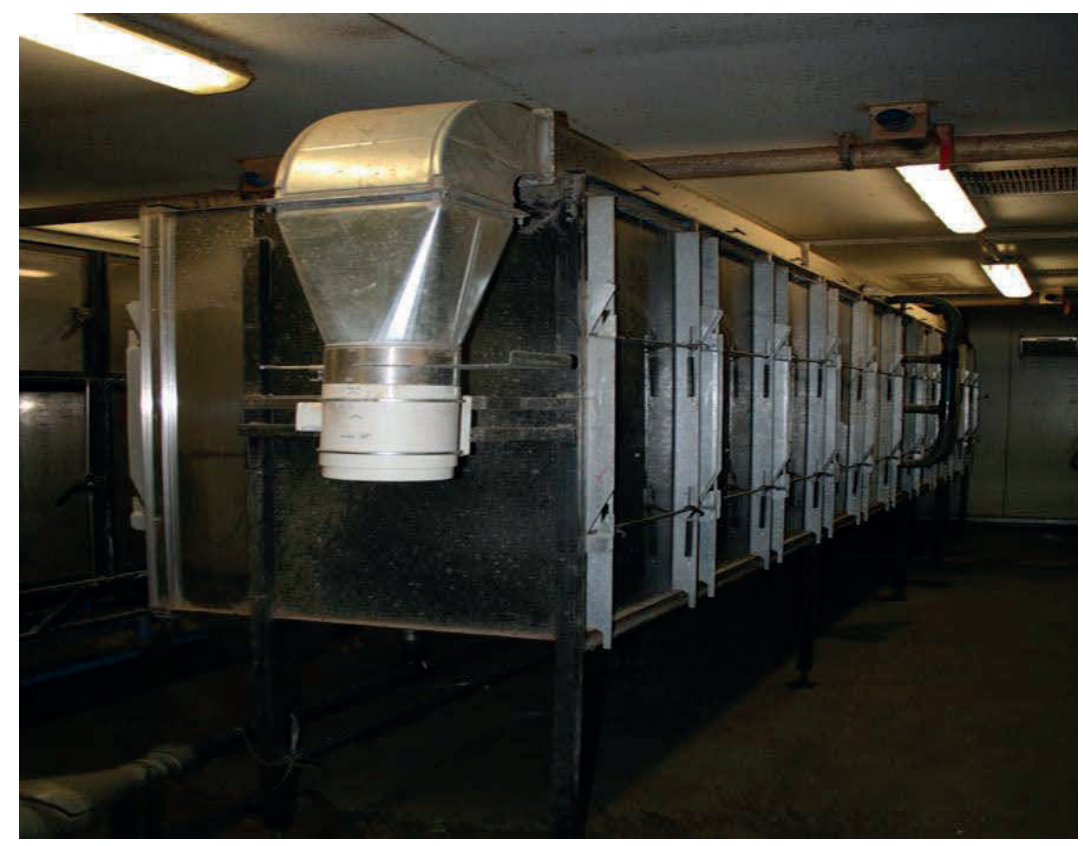

Figure 5. Moth emergence cabinets.

In the moth collection pans adult moths need to be kept immobile. This is a critical procedure to prevent mating in the collection pans as well as the prevention of damage to adult moth's wings. Mated adults or those with damaged wings may have a negative effect on their field performance. However, no set or established temperature range were used during the moth collection, handling, and transport. This led to moths being exposed to temperatures below their critical thermal limits (below $6^{\circ} \mathrm{C}$ ), resulting in poor field recaptures in the warmer months of the season (Boersma and Carpenter 2016; Boersma et al. 2017).

In 2015, new cooling and handling protocols were introduced with a cold chain with a set temperature range from the moth cabinets to the orchard to ensure moths were kept between $6-10^{\circ} \mathrm{C}$, which resulted in better quality and recapture of sterile moths in the field. 


\subsection{Sterilisation Dose}

As soon as the moths reached a required temperature of $10^{\circ} \mathrm{C}$ in the collection pans, they were placed into cardboard boxes $(140 \mathrm{~mm} \times 140 \mathrm{~mm} \times 50 \mathrm{~mm})$ and irradiated with a dose of $150 \mathrm{~Gy}$ (Bloem et al. 2003) in a $20 \mathrm{kCi}{ }^{60} \mathrm{Co}$ source panoramic irradiator. During the 2017-2018 season, the irradiation dose was increased to $200 \mathrm{~Gy}$ to compensate for an apparent reduced sterilisation effect of the 150 Gy dose with reduced dose-rate of the cobalt source. Although the strength of the cobalt source has weakened, and moth exposure time adjusted accordingly, the reason for this decrease in radiation impact is not known and is currently being investigated.

\subsection{Release Methods and Devices}

The irradiated moths are stored in a holding room between $6-8^{\circ} \mathrm{C}$ for approximately 12-24 $\mathrm{h}$, and then transported to orchards or an airfield in a refrigerated vehicle at the same, regulated temperature range.

From 2007 to 2010, sterile moths were released with all-terrain vehicles (ATVs) or "quad bikes", manned by a driver and an assistant responsible for releasing the moths by hand into the trees (Hofmeyr et al. 2015). Later the ATVs were equipped with a release box with a release auger. Although this release method was relatively inexpensive, it had a few disadvantages:

1. Human factor: releasing an accurate and constant number of moths in orchards was not possible.

2. The terrain where some of the orchards are located is rough, making driving while releasing an equal number of sterile moths difficult, leading to inadequate moth distribution.

3. Access to farms was sometimes difficult.

4. Logistical constraints: covering releases twice a week in a valley which stretches more than $100 \mathrm{~km}$ in its length and $60 \mathrm{~km}$ in its width became a logistical constraint; this led to an increasing cost of maintaining the ATV's and preventing breakdowns with a constant challenge of completing the releases in time, versus maintaining the quality of the product.

Since 2010, releases of the moths have progressed from ground releases with ATVs to aerial releases using gyrocopters, and later to fixed-wing aircrafts. Moth releases with fixed-wing Piper Pawnee aircrafts commenced at the end of 2015 but were gradually replaced by helicopters in 2017.

In 2010, Xsit outsourced the releases to a company that used gyrocopters. The release system and holding boxes (hopper) of the sterile moths were slightly modified, and fitted to the gyrocopter, making aerial releases possible. The results obtained were excellent. The recapture rate of the sterile moths increased, while the wild false codling moth population decreased to the lowest levels since the start of the Xsit programme. Unfortunately, after the tragic loss of two pilots, in two separate incidents, the gyrocopters were grounded by the South African Civil Aviation Authority, and releases had to be continued using fixed-wing aircraft. Although the results obtained from the fixed-wing aircraft were comparable to the gyrocopter in certain areas, it had a few disadvantages: 
1. The minimum speed the aircraft flew were significantly higher than the gyrocopters $(160 \mathrm{~km} / \mathrm{h}$ vs. $100 \mathrm{~km} / \mathrm{h})$, resulting in poorer recaptures.

2. The minimum height the aircraft flew were higher than the gyrocopter (160 feet vs. 100 feet), leading to poorer recaptures.

3. Flying in small valleys and mountainous areas were impossible, adding to logistical constrains by filling gaps with ground releases.

4. Quality degradation due to the prop wash of the aircraft (the force of wind generated behind a propeller) causing moths to be blown into a swirl.

Starting the 2017-2018 season, fixed-wing releases were gradually phased out and replaced with small helicopters (R22) to simulate the conditions of gyrocopter releases. This increased efficiency and resulted in sustainable results, contributing to an even greater suppression of wild false codling moth over the past two seasons. Currently the possibility of releasing the sterile insects by unmanned aerial vehicles is being investigated, with the first experimental releases occurring in early 2018. Current aviation legislation in South Africa, in conjunction with costs, still make this venture impossible on a commercial scale.

\section{THE RESULTS AND IMPACT}

The SIT programme for T. leucotreta is governed by phytosanitary requirements, demanding a zero-tolerance level of pest incidence in fruit. The SIT programme is one component of an area-wide approach integrating multiple tactics to mitigate the threat posed by the $T$. leucotreta. This includes obligatory orchard sanitation by growers, with various alternative control measures.

The frequency of sterile male releases varies for each insect species and depends mainly on the survival of the sterile insects in the target area. The ability of sterile insects to survive and remain sexually active as long as possible in the field is essential, and if their longevity declines, the frequency of releases needs to be increased to ensure optimal overflooding ratios at all times (Dowell et al. 2021). The success of the sterile $T$. leucotreta release programme is very much determined by the ability to ensure the release of pre-determined numbers of sterile moths into the orchards that will guarantee a minimum sterile:wild male overflooding ratio of 10:1 (as assessed by trap catches).

In the warmer months, sterile $T$. leucotreta has a shortened life span, therefore requiring two releases of 1000 sterile adults per week. These double releases take place between November to April, while only one release of 2000 sterile adults per week takes place in the cooler months (September to October, and May to June), when the longevity of the moths increases. If the desired overflooding ratio is not achieved, supplementary releases of sterile moths are conducted. Maintaining a continuous optimal overflooding ratio maximises the probability that a wild moth will mate with a sterile moth in the field, thereby resulting in no viable or fertile offspring and an eventual population decline (Carpenter et al. 2004; Hofmeyr et al. 2015). 
Once the wild population has been reduced to such a level that no wild moths are captured on a consistent basis, the release of sterile moths can become the main or sole control method, as is currently the case in numerous citrus orchards in various valleys. Furthermore, the pre- and post-harvest absence of $T$. leucotreta infested fruit, are additional indicators of programme success.

In recent years, the success of the SIT programme is evident by a marked reduction of the wild T. leucotreta populations, in some areas below economic thresholds. The released and wild populations are monitored at weekly intervals, using delta traps baited with the female sex pheromone to attract male moths. Sterile males are differentiated from their wild counterparts by their pink intestines caused by a food dye that is mixed with the larval diet (Hofmeyr et al. 2015).

Commercial results for the three main growing areas currently serviced by the programme, one in the Western Cape, and two in the Eastern Cape, are encouraging. Despite many challenges, the SIT has proven to be a sustainable approach to reduce the occurrence of wild T. leucotreta to well below economic thresholds (results from the recently incorporated Hex River Valley in the Western Cape, and the lower Orange River Valley in the Northern Cape will not be reported here).

\subsection{Olifants River Valley, Western Cape}

The positive achievements of the area-wide programme were evidenced by the progressive increase in the numbers of sterile male $T$. leucotreta moths trapped from 2007 to 2010 (Hofmeyr et al. 2015). In addition, trap catches of wild male adults declined from 13.0 moths per trap per week prior to the sterile moth releases in 2006, to $2.0,0.4$, and 0.1 moths per trap per week in 2012, 2013, and 2014, respectively. In addition, infestation of T. leucotreta in citrus fruit was reduced from $2.6 \%$ in the 2010 2011 season to $0.1 \%$ in 2013 (Barnes et al. 2015; Hofmeyr et al. 2015).

During the 2006-2007 season, i.e. before the start of the area-wide SIT programme, each tree had on average 30 fruit damaged by larvae of the false codling moth. As the SIT programme advanced, and the ratio of sterile to wild adult $T$. leucotreta increased, the average infestation rate declined to only 0.2 damaged citrus fruits per tree per season. The Perishable Products Export Control Board of South Africa reported a substantial reduction of pre-harvest crop losses (Hofmeyr et al. 2015).

Despite this initial success, the number of trapped wild T. leucotreta males increased during the 2010-2011 season, as did crop damage, to an average of 1.56 infested fruits per tree (Fig. 6). However, this was still 95\% lower than the damage level before the release programme started. Higher than normal ambient temperatures experienced during the spring and summer of 2010-2011 were attributed as the cause of the increased wild male captures. This resulted in at least one additional generation, leading to more pressure on the SIT programme resulting in the increased fruit damage. 
Despite this temporary upsurge in wild male catches, the suppression of the wild T. leucotreta population in the SIT-treated areas was restored and progressively improved from 1.5 moths per trap per week in the 2010-2011 season to 0.1 moths per trap per week in the 2015-2016 season (Fig. 6). The gyrocopter accidents coupled with production issues during the 2016-17 season resulted in a slight increase of average wild male catches from 0.3 and 0.4 males per trap per week. This, however, did not result in an increase in fruit infestation. This could be explained by the increased rearing efficiency, resulting in the release of better-quality moths, and the fact that the wild population of T. leucotreta was so low that sterile released adults were more effective, outcompeting the low numbers of the wild population (Hofmeyr et al. 2015).

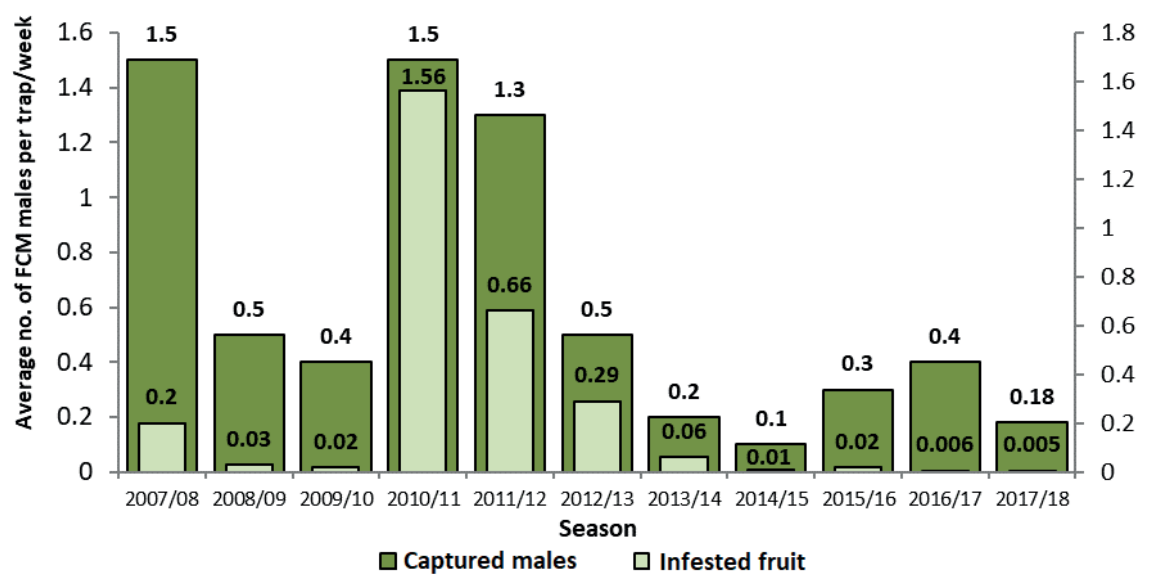

Figure 6. Reduction in numbers of wild $\mathrm{T}$. leucotreta males and infested fruit in sterile insect release areas in the Olifants River Valley, Western Cape Province from 2007-2008 to 20172018 seasons (data obtained from Xsit).

\subsection{Sundays River Valley, Eastern Cape}

Sterile moth releases were initiated in the Sundays River Valley in 2011-2012, and since then the density of the wild $T$. leucotreta population has progressively declined with successive seasons, resulting in less infested fruit (Fig. 7). There was, however, an increase in the average number of trapped wild males in 2013-2014, but this was due to areas with historically high population densities being added to the SIT programme.

A similar trend of higher wild trap catches was seen in the Sundays River Valley in 2016-2017 due to challenges experienced in the mass-rearing facility. During the 2017-2018 season wild false codling moth catches decreased to only 1 wild male per trap and 0.02 infested fruit per tree respectively, the lowest since the start of the SIT programme. 


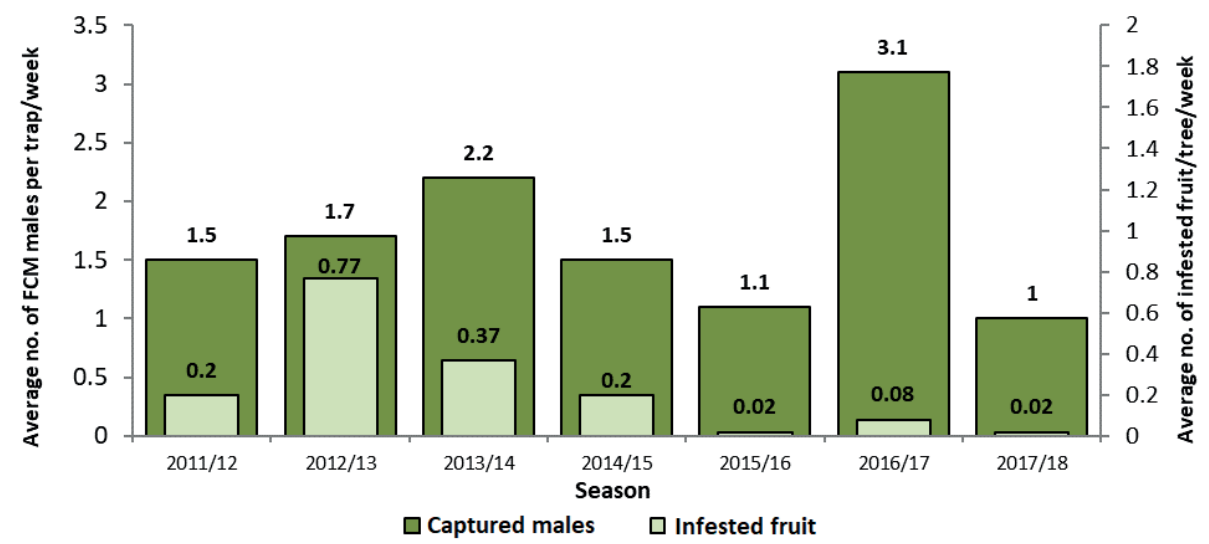

Figure 7. Reduction in numbers of wild T. leucotreta males and infested fruit in the Sundays River Valley, Eastern Cape Province from 2011-2012 to 2017-2018, as a result of sterile insect releases (data obtained from Xsit).

\subsection{Gamtoos River Valley, Eastern Cape}

The natural population of T. leucotreta was much lower in the Gamtoos Valley as compared to the other areas, causing much less crop damage. This was evidenced by much lower trap catches of wild males during the first season of sterile moth releases in comparison to the other areas. As a result, the SIT programme was able to reduce the wild moth population density and the number of infested fruits within the first release season (2014-2015).

In the following season, wild males were suppressed to such a low level that basically no infested fruit were recorded for the entire season, while a slight increase was recorded in the 2016-2017 season for the same reasons mentioned above (challenges at the rearing facility) (Fig. 8).

\section{THE REASONS FOR SUCCESS}

This AW-IPM programme with a sterile male release component against the $T$. leucotreta has had a significant impact on the citrus industry in South Africa; securing exports to the rest of the world in a sustainable manner. The success of the programme can be attributed to the following factors:

1. Single crop industry: The citrus trade, unlike other fruit sectors such as the deciduous fruit industry, is a single crop industry led by the Citrus Growers Association. This results in easier decision-making, management, and funding, since all stakeholders have the same vision. This played a significant role in the success of the programme as it was an industry-driven project to secure sustainable citrus exports for the growers. 


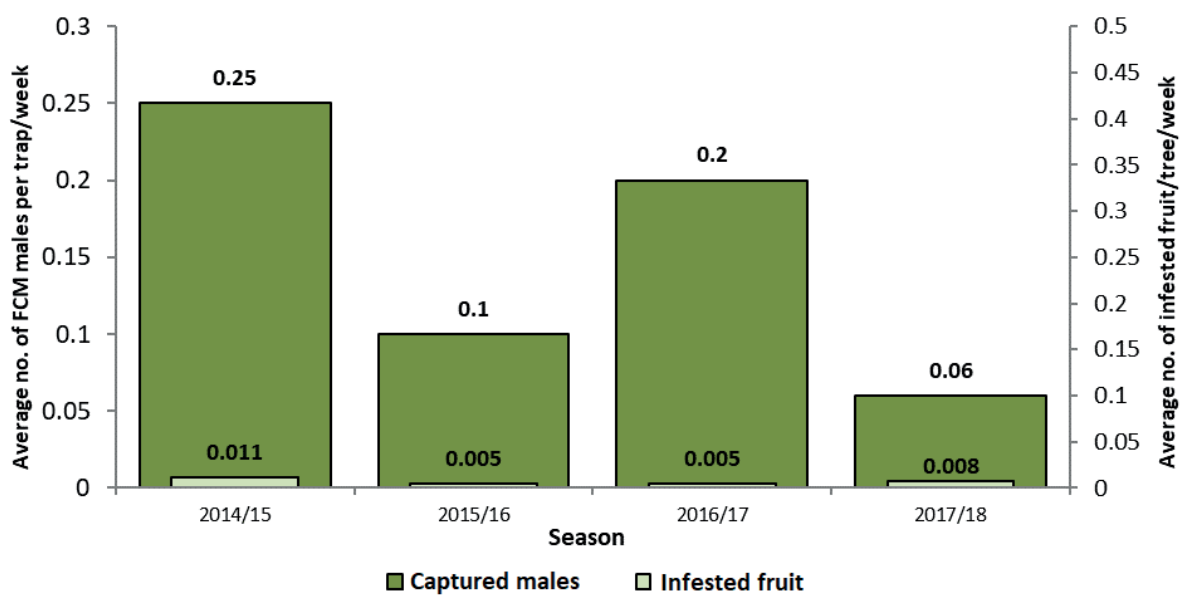

Figure 8. Reduction in numbers of wild $\mathrm{T}$. leucotreta males and infested fruit in the Gamtoos River Valley, Eastern Cape Province from 2014-2015 to 2017-2018 seasons, as a result of sterile insect releases (data obtained from Xsit).

2. Area-wide integration of suppression methods: The SIT programme was managed as part of an area-wide programme. Xsit did not only take responsibility of both the monitoring of wild T. leucotreta and infestation, but also played a significant role in the monitoring of sanitation practises and the treatment of hot spots (an area with a high wild $T$. leucotreta population), in conjunction with other integrated pest control practises.

3. Management to ensure sustainable sterile moth production: Well-experienced and capable management was in place which ensured sustainable production of sterile insects, while the shareholders of the programme were also industry-related individuals, which ensured that the interests of the programme were always wellmanaged.

4. Support of farmers and industry: Most farmers were in favour of the programme, understanding the advantages of the AW-IPM approach. However, education and training were provided throughout the programme to ensure farmers were kept informed about industry-related matters.

5. Phytosanitary regulations: Since T. leucotreta is a regulated quarantine pest, governed by phytosanitary regulations in line with a systems approach (FAO 2017) for the controlling of the pest for export purposes, a zero-tolerance policy is enforced. Although there are several choices of control measures for T. leucotreta under the systems approach, it encouraged farmers to take part in the SIT programme if they wanted to export their fruit.

6. In-house research: Xsit employed its own researchers, constantly exploring better means of rearing, processing, and releasing insects, staying informed of the newest technology. 
7. Set protocols and procedures: The use of standard protocols and procedures combined with continuous training of employees are essential for the efficient rearing of insects. The correct handling and distribution of insects are also essential to ensure good quality of insects in the field. Maintaining a cold chain proved essential to prevent damage to the sterile insects during transport, while the proper handling temperature has to be selected as this affects the competitiveness of the adults in the field (Boersma and Carpenter 2016).

\section{FUTURE PLANS}

The need for AW-IPM programmes with a SIT component to manage T. leucotreta in other countries where this pest is present, has become more apparent. Export crops such as avocados in Angola, and chilies in Kenya may also require the use of a SITbased AW-IPM in the future to deal with this polyphagous pest.

Meanwhile, many export crops in South Africa, such as table grapes, stone fruits, and citrus grown in other regions are anticipating the introduction of the SIT. Xsit currently services 14000 ha of the 70000 ha of citrus in South Africa, while 4000 ha of table grapes are already enrolled in the programme. This indicates that there is scope for integration and expansion of this valuable technology to production areas not yet under this area-wide pest control method.

The potential of $T$. leucotreta becoming a major invasive pest in different countries globally is a reality, representing a threat to agriculture and food security. With the SIT now developed for this pest, if invasive false codling moth outbreaks are detected early, efficient integration of the SIT on an area-wide basis will allow eliminating them in an effective and environment-friendly way.

\section{REFERENCES}

Barnes, B. N., J. H. Hofmeyr, S. Groenewald, D. F. Conlong, and M. Wohlfarter. 2015. The Sterile Insect Technique in agricultural crops in South Africa: A metamorphosis .... but will it fly? African Entomology 23: 1-18.

Bloem, S., J. E. Carpenter, and J. H. Hofmeyr. 2003. Radiation biology and inherited sterility in false codling moth (Lepidoptera: Tortricidae). Journal of Economic Entomology 96: 1724-1731.

Boersma, N., and J. E. Carpenter. 2016. Influence of holding temperature and irradiation on field performance of mass-reared Thaumatotibia leucotreta (Lepidoptera: Tortricidae). Florida Entomologist 99 (Special Issue 1): 215-221.

Boersma, N., L. Boardman, M. Gilbert, and J. S. Terblanche. 2017. Sex-dependent thermal history influences cold tolerance, longevity and fecundity in false codling moth Thaumatotibia leucotreta (Lepidoptera: Tortricidae). Agriculture and Forest Entomology 20: 41-50.

Carpenter, J. E., S. Bloem, and J. H. Hofmeyr. 2004. Acceptability and suitability of eggs of false codling moth (Lepidoptera: Tortricidae) from irradiated parents to parasitism by Trichogrammatoidea cryptophlebiae (Hymenoptera: Trichogrammatidae). Biological Control 30: 351-359.

Dowell, R. V., J. Worley, P. J. Gomes, P. Rendón and R. Argilés Herrero. 2021. Supply, emergence, and release of sterile insects, pp. 441-484. In V. A. Dyck, J. Hendrichs, and A. S. Robinson (eds.), Sterile Insect Technique - Principles and practice in Area-Wide Integrated Pest Management. Second Edition. CRC Press, Boca Raton, Florida, USA.

(FAO) Food and Agriculture Organization of the United Nations. 2017. The use of integrated measures in a systems approach for pest risk management. International Standard for Phytosanitary Measures (ISPM) No. 14. International Plant Protection Convention. FAO, Rome, Italy. 
Hendrichs, J., P. Kenmore, A. S. Robinson, and M. J. B. Vreysen. 2007. Area-Wide Integrated Pest Management (AW-IPM): Principles, practice and prospects, pp. 3-33. In M. J. B. Vreysen, A. S. Robinson, and J. Hendrichs (eds.), Area-wide control of insect pests. From research to field implementation. Springer, Dordrecht, The Netherlands.

Hofmeyr, J. H., and K. L. Pringle. 1998. Resistance of false codling moth, Cryptophlebia leucotreta (Meyrick) (Lepidoptera: Tortricidae), to the chitin synthesis inhibitor, triflumuron. African Entomology 6: 373-375.

Hofmeyr, J. H., and J. Pretorius. 2010. Development of a device to collect mass-reared false codling moth, Thaumatotibia leucotreta (Meyrick) (Lepidoptera: Tortricidae), in a commercial insectary. African Entomology 18: 374-378.

Hofmeyr, J. H., J. E. Carpenter, and S. Bloem. 2005. Developing the Sterile Insect Technique for Cryptophlebia leucotreta (Lepidoptera: Tortricidae): Influence of radiation dose and release ratio on fruit damage and population growth in field cages. Journal of Economic Entomology 98: 1924-1929.

Hofmeyr, J. H., J. E. Carpenter, S. Bloem, J. P. Slabbert, M. Hofmeyr, and S. S. Groenewald. 2015. Development of the Sterile Insect Technique to suppress false codling moth Thaumatotibia leucotreta (Lepidoptera: Tortricidae) in citrus fruit: Research to implementation (Part 1). African Entomology 23: $180-186$.

Hofmeyr, J. H., M. Hofmeyr, V. Hattingh, and J. P. Slabbert. 2016a. Postharvest phytosanitary disinfestation of Thaumatotibia leucotreta (Lepidoptera: Tortricidae) in citrus fruit: Determination of ionising radiation and cold treatment conditions for inclusion in a combination treatment. African Entomology 24: 208-216.

Hofmeyr, J. H., V. Hattingh, M. Hofmeyr, and J. P. Slabbert. 2016b. Postharvest phytosanitary disinfestation of Thaumatotibia leucotreta (Lepidoptera: Tortricidae) in citrus fruit: Validation of an ionising radiation and cold combination treatment. African Entomology 24: 217-224.

Klassen, W., and M. J. B. Vreysen. 2021. Area-Wide Integrated Pest Management and the Sterile Insect Technique, pp. 75-112. In V. A. Dyck, J. Hendrichs, and A. S. Robinson (eds.), Sterile Insect Technique - Principles and practice in Area-Wide Integrated Pest Management. Second Edition. CRC Press, Boca Raton, Florida, USA.

Moore, S. D. 2002. The development and evaluation of Cryptophlebia leucotreta granulovirus (ClGV) as a biological control agent for the management of false codling moth, Cryptophlebia leucotreta, on citrus. Ph.D. thesis, Rhodes University, Grahamstown, South Africa. 308 pp.

Moore, S. D., G. I. Richards, C. Chambers, and D. Hendry. 2014. An improved larval diet for commercial mass rearing of the false codling moth, Thaumatotibia leucotreta (Meyrick) (Lepidoptera: Tortricidae). African Entomology 22: 216-219.

Oates, C. G. 1997. Towards an understanding of starch granule structure and hydrolysis. Trends in Food Science and Technology 8: 375-382.

Wang, S., C. Li, L. Copeland, Q. Niu, and S. Wang. 2015. Starch retrogradation: A comprehensive review. Comprehensive Reviews in Food Science and Food 14: 568-585. 